\title{
Analysis of free fatty acids in plasma for the prognosis of cardiovascular events after acute myocardial infarction: study protocol for prospective, observational, single-center cohort trial.
}

\section{Weiwei Chen}

China-Japan Union Hospital of Jilin University

Shuang Wang

China-Japan Union Hospital of Jilin University

Xu Yan

China-Japan Union Hospital of Jilin University

Huan Sun

China-Japan Union Hospital of Jilin University

Ping Yang ( $\nabla$ pyang@jlu.edu.cn )

China-Japan Union Hospital of Jilin University https://orcid.org/0000-0001-7960-6248

\section{Research Article}

Keywords: Free Fatty Acid, Acute Myocardial Infarction, Prognosis, study protocol

Posted Date: July 29th, 2021

DOI: https://doi.org/10.21203/rs.3.rs-761793/v1

License: (c) (1) This work is licensed under a Creative Commons Attribution 4.0 International License. Read Full License 


\section{Abstract}

- Background

- It is not currently known whether free fatty acids can be used as predictors for the prognosis of patients with acute ST-segment elevation myocardial infarction. The results of many studies on the correlation between free fatty acids and the prognosis of patients with acute myocardial infarction are inconsistent. The purpose of this clinical study is to determine the predictive effect of different free fatty acids in the plasma on cardiovascular events within the 12 months after a patient suffers an acute ST-segment elevation myocardial infarction.

- Methods

- This study is a prospective, observational, single-center cohort study. After enrollment, all participants will receive standard treatment and will be tested for 31 kinds of plasma free fatty acids. The patients will be followed up 2, 6, and 12 months after discharge. The primary endpoint is the compound cardiovascular events from onset to 12 months, including the cumulative incidence of all-cause death, heart failure, or coronary revascularization events. According to the power and sample size calculation, it will be necessary to enroll 747 patients in this study. After follow-up, data will be collected and analyzed to explore the predictive effect of different free fatty acids in plasma on the prognosis of acute elevated ST-segment myocardial infarction, and the risk factors of the endpoint events within 12 months.

- Discussion

- This study will provide new insightful evidence of serum indicators indicating the 12-month cardiovascular events of acute ST-segment elevation myocardial infarction.

Trial registration: This study was retrospectively registrated at Chinese Clinical Trail Registry (https://www.chictr.org.cn/) on 14th July,2021. Registration number: ChiCTR2100048731.

\section{Introduction}

\section{Background and rationale $\{6 a\}$}

Acute myocardial infarction (AMI) is still the leading cause of cardiovascular death ${ }^{1}$ According to epidemiological reports, there are more than 7 million cases of acute myocardial infarction every year worldwide ${ }^{2}$. The high mortality and disability rate of the disease increases the burden on patients and the health system. Therefore, the identification of prognostic factors is significant to improve patient outcome.

Free fatty acids (FFA) provide $60-70 \%$ of adenosine triphosphate needed for myocardial metabolism, but the $\beta$-oxidation of FFA consumes more oxygen than glycolysis. When coronary artery occlusion occurs, cardiomyocytes are unable to use FFA due to the insufficient oxygen supply. The conversion to using 
glucose as a substrate, leads to a decrease in FFA utilization, resulting in aggravation of the cells due to the excess FFA. The levels of FFA in the serum of patients with AMI is increased, and this has the potential to be used as a biochemical marker. The high level of FFA reflects the severity of myocardial ischemia and myocardial necrosis ${ }^{3}$. This may be the result of the pathophysiological mechanism of AMI, rather than the cause.

In recent years, more and more studies have confirmed the correlation between FFA and heart disease. A cross-sectional study showed a positive correlation between plasma FFA and the risk of coronary heart disease ${ }^{4}$. In addition, a clinical study ${ }^{5}$ involving 28 male patients with coronary heart disease and 25 male controls showed that plasma FFA at 8 hours after dietary fat load was shown to correctly identify patients with coronary heart disease. However, in a prospective study, FFA concentrations were not associated with coronary heart disease death after adjusting for traditional risk factors ${ }^{6}$. The multivariate model in this study included blood pressure variables, which may be a potential mediator between FFA and coronary heart disease. Schrieks et al., ${ }^{7}$ found that the total FFA level is a predictor of poor prognosis in acute coronary syndrome (ACS), but it is not clear whether FFA is the main predictor of poor prognosis in AMI. The contradictory phenomenon of the results may be since these studies only discussed the relationship between the total plasma FFA and the prognosis of coronary heart disease. Different kinds of FFA may play different roles in the pathogenesis of AMI. Eicosapentaenoic acid (EPA) and a-linolenic acid (ALA), are anti-inflammatory factors and therefore protective factors for the prognosis of $\mathrm{AMI}^{8}$. Docosahexaenoic acid (DHA) seems to show differing results between various studies ${ }^{8,9}$.c may be an adverse prognostic factor for AMI due to its pro-inflammatory effect ${ }^{10}$. It may be limiting to simply analyze the prognosis based on the total FFA and AMI alone. Therefore, the analysis of various components of the total free fatty acids may help to better determine the relationship between free fatty acids and the prognosis of AMI. Herein we aim to explore the correlation between total FFA, the different types of FFA, and the prognosis of AMl; and to determine whether it can assist in the prognosis of AMI. We will use highly sensitive high-performance liquid chromatography (HPLC) to determine the various FFA in the plasma of patients after AMI to determine whether it can be used as an independent predictor of AMI prognosis, and to provide insightful suggestions for clinical work.

\section{Objectives $\{7\}$}

The main hypotheses of this study is that 31 kinds plasma levels could predict the risk of cardiovascular events in patients 12 months after AMI.

The secondary hypotheses of this study that 31 kinds plasma levels could predict the risk listed below in patients 12 months after AMI:

- all-cause death;

- heart failure; 
- coronary artery revascularization;

- non-fatal myocardial infarction;

- non-fatal stroke,

- malignant arrhythmia,

- heart failure,

- unstable angina pectoris,

- and target vessel revascularization.

\section{Trial design $\{8\}$}

This study is a prospective, observational, single-center study for acute myocardial infarction. All subjects will give informed consent before participating in the study according to the Helsinki Declaration's ethical guidelines. Participants will be recruited from the Department of Cardiology, China-Japan Union Hospital, Jilin University. All potential participants will receive information about the trial, and researchers and potential participants will first need to complete the informed consent process. The researchers will provide informed consent to each potential participant and then confirm that participation in the study is voluntary. Refusal to participate in this study will not affect the quality of medical care received by the patients. Subsequently, the lead researcher or researcher will confirm whether potential participants meet the inclusion criteria. After enrollment, all participants will receive standard treatment and will be tested for 31 kinds of plasma free fatty acids. The patients will be followed up 2, 6, and 12 months after discharge. At the time of enrollment, consent for longitudinal follow-up via telephone and collection of future medical records is obtained, as is information for emergency contacts to prevent loss to follow-up. Follow-up occurs via centralized telephone interviews by trained personnel. This centralized approach maximizes the consistency and quality of data collection. During 2-, 6-, and 12-months follow-up, participants are asked to report medications and adherence, clinical outcomes defined in our study, and the medical record in the latest time. All researchers involved in the project will receive unified questionnaire training to reduce data bias. All authors will be fully responsible for the design, implementation, analysis, drafting, and editing of the paper and the final content of the article.

\section{Methods: Participants, Interventions And Outcomes}

\section{Study setting $\{9\}$}

This study is a prospective, observational, single-center study for acute myocardial infarction. Data would be collected in China-Japan Union Hospital. 
Admission criteria:

Patients with acute ST-segment elevation myocardial infarction will be admitted according to the diagnostic criteria recommended in 2017 by the European Society of Cardiology (ESC) guidelines for STsegment elevation myocardial infarction ${ }^{12}$ :

(1) Clinical symptoms of myocardial ischemia; (2) The electrocardiogram showed new myocardial ischemia changes and new ST-segment elevation changes of the left bundle branch block. (3) Pathological Q wave appeared in Electrocardiograph (ECG).

\section{Exclusion criteria:}

(1) Refusal to participate in the study; (2) Refusal to cooperate with the collection of information, or unwilling to follow up; (3) Age over 90 years old; (4) Thyroid dysfunction (thyrotropin < the lower limit of the normal value, or $>1.5$ times the upper limit of the normal value, free thyroxine T4 level is outside the

normal range); (5) Severe renal insufficiency (estimated glomerular rate filter $<20 \mathrm{ml} / \mathrm{min} / 1.73 \mathrm{~m}^{2}$ ); (6) Severe liver insufficiency (aspartate aminotransferase or alanine aminotransferase $>3$ times the normal value); (7) Transplant of any major organ; (8) Pregnancy or lactation; (9) Suffering from mental illness; the presence of any infectious or systemic inflammatory disease; (10) Hematological disease; (11) Tumor; (12) Metabolic disease.

\section{Who will take informed consent? $\{26 \mathrm{a}\}$}

Professional clinicians from China-Japan Union hospital will make preliminary diagnoses and evaluate emergency patients. Those patients who meet the criteria must sign an informed consent form before the collection of any data, including medical records, contact information, emergency contacts and other information.

\section{Additional consent provisions for collection and use of participant data and biological specimens $\{26 \mathrm{~b}\}$}

This informed consent is to solicit your opinions on preserving and using your hospital diagnosis and treatment information and the remaining blood and body fluids during the diagnosis and treatment process. Specific instructions are as follows:

\section{About patients:}

Allow researchers to view, collect, and use information about your illness. The collection of samples is carried out after the bedside blood collection and other medical treatment, which does not harm your body, does not affect or damage your definitive diagnosis and treatment and does not harm your health. You will not have to pay any additional fees and will not benefit financially in any way from this study. To 
protect personal information and privacy, no other person is allowed to know the information related. Patients may voluntarily provide samples and may cancel and withdraw the consent at any time; Even if the patients refuse to give the sample, it will not affect all your standard diagnosis and treatment process.

\section{About researchers:}

Researchers will keep these samples and clinical information to use in this study in the future.

Even if the patients refuse to provide the sample, we will still provide him/her with the same medical services as the sample provider. All samples will be used up anonymously. If they need to be discarded, the relevant personal information will be destroyed at the same time. Researchers will publish the results of our research for the benefit of medical development without disclosing the personal data of the sample providers. Intellectual property derived from research results belongs to the research unit and the researcher, not to the sample provider.

\section{Interventions}

\section{Explanation for the choice of comparators $\{6 \mathrm{~b}\}$}

Not applicable. This study is an observational cohort study. Grouping is determined by whether the subjects had a primary or secondary endpoint event during the follow-up period after admission. No randomization procedure or interventions would be used.

\section{Intervention description $\{11 \mathrm{a}\}$}

Not applicable.

\section{Criteria for discontinuing or modifying allocated interventions $\{11 \mathrm{~b}\}$}

Not applicable.

\section{Strategies to improve adherence to interventions $\{11 \mathrm{c}\}$}

Not applicable. 
Not applicable.

\section{Provisions for post-trial care $\{30\}$}

Not applicable.

\section{Outcomes $\{12\}$}

\section{Primary endpoint}

The main endpoint of this study will be the compound endpoint of cardiovascular events within 12 months of onset, including the cumulative incidence of all-cause death, heart failure events, or coronary revascularization events.

Definition of all-cause death. death within 12 months after onset, regardless of cause. If death occurs before discharge, it will be subdivided into cardiac death and non-cardiac death according to physical and chemical examination, clinical diagnosis, and other information. After discharge, death found by follow-up is difficult to determine and so will be classified as all-cause death.

Definition of heart failure the occurrence of heart failure within 12 months after the onset of the disease. Clinicians will assess in-hospital heart failure events based on new heart failure symptoms, new objective evidence of heart failure, and initial intensive treatment specifically for heart failure. Heart failure found by the follow-up after discharge will be defined as the patient having new symptoms of heart failure, has new objective evidence of heart failure, or is treated with anti-heart failure drugs. The criteria for judging heart failure are the need to meet at least two symptoms and at least one laboratory standard or objective index.

Symptoms of heart failure include (1) Dyspnea (dyspnea, dyspnea in expiratory phase, paroxysmal nocturnal dyspnea, shortness of breath in supine position), (2) Decreased exercise tolerance (due to symptoms of dyspnea or fatigue), or reduced ability to perform large-scale skeletal muscle dynamic exercise, and (3) Severe end-organ failure (brain, kidney, liver, abdomen, or deterioration of symptoms, such as fever, dizziness, syncope, confusion, restlessness, cognitive decline, nausea, abdominal distension, abdominal discomfort, abdominal tenderness, chills relief, limb discoloration, extreme reduction of jaundice in urine). The New York heart function classification was III and IV. Class III is defined as a marked limitation of physical activity; comfortable at rest, but less than ordinary activity causes fatigue, palpitations, or dyspnea. Class IV is defined as being unable to carry out any physical activity without discomfort; symptoms of cardiac insufficiency at rest. 
The signs of heart failure are as follows: (1) Peripheral edema (edema of lower extremities, compression of ankles, legs, thighs, abdomen, upper limbs, and facial edema), (2) Abdominal distension or increased ascites (in the absence of primary liver disease), (3) Pulmonary rales, pulmonary edema, pleural effusion, (4) Elevated jugular pressure and hepatic-jugular reflux, and (5) Heart auscultation gallop rhythm.

Objective evidence standard. (1) Cardiac color ultrasound: ejection fraction is less than $45 \%$, (2) The concentration of plasma BNP or NT-proBNP increased, BNP > $500 \mathrm{pg} / \mathrm{mL}$ or NT-proBNP $>2000 \mathrm{pg} / \mathrm{mL}$, (3) The walking distance of fewer than 150 meters in 6 minutes indicates severe cardiopulmonary insufficiency. The walking distance of 150-450 meters shows moderate cardiopulmonary insufficiency, and the walking distance of more than $\mathbf{4 5 0}$ meters shows mild cardiopulmonary insufficiency.

The definition of coronary artery revascularization: coronary revascularization therapy is performed again because of coronary artery stenosis in the hospital or after discharge. If vascular lesions are evaluated for the first time in the hospital, the lesions that need to be treated in the hospital are not recorded. The site of coronary artery stenosis can include original stenosis and new or progressive stenosis of non-target diseases. Revascularization therapy includes balloon dilatation, thrombus aspiration, stent implantation, and rotational grinding of coronary plaques.

\section{Secondary endpoints}

Secondary endpoints include all-cause death, non-fatal myocardial infarction, non-fatal stroke, malignant arrhythmia, heart failure, unstable angina pectoris, and target vessel revascularization. The above endpoints are defined according to 2014 American College of Cardiology (ACC) and American Heart Association (AHA) key data elements and definitions for cardiovascular endpoints in clinical trials ${ }^{11}$.

\section{Participant timeline $\{13\}$}

Figure 1 shows the timeline of our study.

\section{Sample size $\{14\}$}

Sample size calculation is based on the main endpoint of this study: the compound endpoint of cardiovascular events within 12 months of onset, including the cumulative incidence of all-cause death, heart failure, and coronary revascularization events. We will use an overall Class I error of 0.05 , and the risk ratio of the composite research endpoint is assumed to be 0.8 . According to the results of OMEGA Study ${ }^{13}$ and CHINA PEACE ${ }^{14}$ studies, the incidence of all-cause death in patients with ST-segment elevation myocardial infarction was $2.7 \%$ and $3.7 \%$ within one year, and the incidence of compound events was about $18.2 \%$. It is estimated that the total incidence of events at the main endpoint of this study will be approximately $19.9 \%$. There are many kinds of free fatty acids, and different free fatty acids have different effects on the prognosis of AMI. We will calculate the sample size of this study according 
to the literature data of total FFA, EPA, and ALA. Shrieks et al., ${ }^{14}$ found that a total FFA level of 7 was a predictor of poor prognosis in ACS, with an Hazard Ratio (HR) of 1.12. EPA and ALA are protective factors for the prognosis of AMI. It was shown that the HR of EPA to MACE events in patients with AMI was $0.76^{8}$. The HR value of ALA for all-cause death was 0.65 . However, DHA seems to show different results in different studies. The relationship between other free fatty acids and the prognosis of AMI is not clear and consequently the sample size is estimated based on the above literature data, and the lost follow-up rate is estimated to be $20 \%$. Power and Sample Size software will be used for calculation, and the estimation is based on the literature ${ }^{15}$. The sample size estimated by total FFA, EPA, and ALA is 747, 23, and 609, respectively. The process is shown in Table 1, and the final estimated sample size is 747.

\section{Recruitment $\{15\}$}

This study was conducted in the Department of Cardiovascular Medicine, China-Japan Union Hospital of Jilin University. Suppose the cardiovascular physician encounters a suitable candidate during the diagnosis and treatment of acute chest pain in the Emergency Room (ER). In that case, the study physician will be notified to conduct further screening and initiate informed consent. The number of emergency patients in the China-Japan Union Hospital of Jilin University is large enough to guarantee adequate participant enrolment.

\section{Assignment of interventions: allocation}

\section{Sequence generation $\{16 a\}$}

Not applicable.

\section{Concealment mechanism $\{16 \mathrm{~b}\}$}

Not applicable.

\section{Implementation $\{16 \mathrm{c}\}$}

Not applicable.

\section{Assignment of interventions: Blinding}




\section{Who will be blinded $\{17 \mathrm{a}\}$}

Not applicable.

\section{Procedure for unblinding if needed $\{17 \mathrm{~b}\}$}

Not applicable.

\section{Data collection and management}

\section{Plans for assessment and collection of outcomes $\{18 \mathrm{a}\}$}

Professional clinicians will make preliminary diagnoses and evaluate emergency patients. Those patients who meet the criteria must sign an informed consent form before the collection of any data, including medical records, contact information, emergency contacts, and other information, to prevent loss of follow-up. At the same time as patients undergo standardized treatment, blood samples will be taken for free fatty acid spectrum determination by high-performance liquid chromatography-mass spectrometry. Measurements will be collected for total free fatty acids, total $\Omega 3$, total $\Omega 6$, total monounsaturated fatty acids, total polyunsaturated fatty acids, triene/tetraene, $\Omega 3 / \Omega 66$, lignocerric acid, nervonic acid, behenic acid, erucic acid, docosatetraenoic acid (DTA), docosapentaenoic acid (DPA), docosahexaenoic acid (DHA), arachidic acid, eicosanoic acid, mead acid, eicosadienoic acid, arachidonic acid (AA), eicosapentaenoic acid (EPA), stearic acid, oleic acid, linoleic acid, linolenic acid, palmitic acid, palmitoleic acid, myristic acid, myristoleic acid, laurric acid, and decanoric acid. On the second day, the baseline questionnaire data will be collected by trained personnel through face-to-face interviews, to maximize the data collection, implementation, and quality. The content of the face-to-face interview is shown in supplementary material. When the patient is discharged from the hospital, the researchers will take the medical records for filing. The information includes all medical records, results of biochemical blood tests, images of coronary angiography and surgical records, blood-free fatty acid test results, heart ultrasound results, ECG results, data structured processing and registration by the researchers if the patient has related clinical events during hospitalization. Trained doctors will use the standard definition of clinical events to collect and judge medical records.

\section{Plans to promote participant retention and complete follow-up $\{18 \mathrm{~b}\}$}

Out-of-hospital follow-up will be conducted in 2, 6, and 12 months through centralized telephone interviews. Participants will be asked to report on quality of life, drug compliance, medical records, and 
descriptions of related events. A professional clinician will assess the definition of the events after the description.

\section{Data management $\{19\}$}

The trained researchers are responsible for recording the baseline information and medical records. The follow-up information would be recorded in the process during the follow-up telephone. After data entry is complete, Data monitoring committee (DMC) will randomly audit $20 \%$ of the medical records and monitor the summary's quality to ensure that the overall variable accuracy is more than $98 \%$.

\section{Confidentiality \{27\}}

When a participant takes informed consent, all data records will be replaced by a participants' unique number to protect their privacy. When the trial is over, the published data set will not contain their names, contact details, and other private information. Data entry, review, and proofreading will be based on the participants' number.

\section{Plans for collection, laboratory evaluation and storage of biological specimens for genetic or molecular analysis in this trial/future use $\{33\}$}

\section{Use of blood samples:}

For each enrolled participant, the test of 31 free fatty acids is described in Plans for Assessment and Collection of Outcomes and any tests necessary to ensure participants' safety. Including virology testing, testing for COVID-19, etc. This testing aims to ensure that analytical methods produce valid data throughout the study. It may also include, but is not limited to, investigations with unexpected results, inprocess analysis of samples, and method transfer and comparability analysis. Our research team may conduct additional tests on the remaining samples to study and better understand free fatty acid products or markers of cardiovascular disease and molecular characterization. The results of these analyses will be recorded and maintained but may not be reported as part of the study.

\section{Laboratory evaluation}

After the informed consent form, the blood samples were collected before the operation, and the plasmafree fatty acids were tested. The rest of the blood sample will be sent to the cardiovascular laboratory in an icebox immediately. In the laboratory, the blood was centrifuged at $3000 \mathrm{rpm}$ at $4^{\circ} \mathrm{C}$ for $10 \mathrm{mins}$. After 
centrifugation, the plasma and white cell layers were separated into tubes. The patient information and tube location information were electronically recorded and stored in the $-80^{\circ} \mathrm{C}$ refrigerator.

\section{Biomarkers and genetic studies}

Biomarkers are objectively measured to evaluate biological and pathophysiologic processes. Research techniques will be further developed in the future, so it is difficult to determine which biomarker should be tested at this stage. If markers can be correlated with the data in this study, they may provide prognostic indicators for patients with acute ST-segment elevation myocardial infarction in the future. Genetic studies can better reveal the distribution of genes in a population, disease susceptibility, and other related issues. The serum and leukocyte layers stored in this study may be tested for biomarkers and genetic material in the future.

\section{Storage and destruction of samples}

The serum and white blood cells from the remaining blood samples in the study will be preserved until 10 years after the end of the study, which will be used to study scientific problems related to cardiovascular disease, metabolic disease, inflammation and so on. By contacting and authorizing the key researchers, the subjects retained the right to destroy the sample material at any time. The researcher is the exclusive owner of the derivative of any data, discovery, or sample material source. The researchers destroyed the samples centrally when the storage period arrived.

\section{Statistical methods}

\section{Statistical methods for primary and secondary outcomes $\{20 \mathrm{a}\}$}

Participants will be divided into 'event' group and 'non-event' group according to whether the main endpoint event occurred. The baseline levels of all hospitalization data between the two groups will be compared. The continuous data of normal distribution is expressed by mean \pm standard deviation (SD). In contrast, the skewed distribution data is expressed by median [quartile], and the classified data is expressed by $\mathrm{n}(\%)$. The students' test will be used to compare the difference of continuous data of normal distribution between the two groups. The Mann-Whitney test will be used to compare the variables of skewed distribution, and the chi-square test used to compare the difference of rates between the two groups. Pearson or Spearman correlation coefficients determine the correlation between the two variables. The levels of serum-free fatty acids in patients with 'non-events' and 'events' will be compared, and various free fatty acids analyzed by cluster analysis. After adjusting for age, gender, history of hypertension, diabetes, cerebrovascular disease, heart failure, myocardial infarction, glomerular filtration rate, triglyceride, total cholesterol, left ventricular ejection fraction, and other confounding factors, multivariate analysis will be carried out. The predictive role of each FFA in the prognosis of AMI will be determined by Cox survival regression. The results will be expressed as Hazzard ratio (HR) and the 
corresponding 95\% confidence interval (CI). SPSS software (SPSS 25.0, Chicago, Illinois) will be used for statistical analysis.

\section{Interim analyses $\{21 \mathrm{~b}\}$}

In this study, there are no planned interim analysis or termination rules. This study is observational. All participants receive standard treatment. There are no interventions in this study, generally do not terminate the trial in advance.

\section{Methods for additional analyses (e.g. subgroup analyses) \{20b\}}

According to the data collected and the relationship between plasma FFA and the prognosis in patients with AMI, researchers may conduct gender subgroup analyses to see whether the plasma FFA predicts the cardiovascular event differently in gender. Meanwhile, researchers would explore the influence factor of plasma FFA.

\section{Methods in analysis to handle protocol non-adherence and any statistical methods to handle missing data $\{20 \mathrm{c}\}$}

\section{The definition of withdrawal:}

Participants signed the informed consent form and selected the cases required to withdraw during the follow-up after selecting qualified participants for the study.

\section{The definition of elimination:}

Participants signed an informed consent form to enter the study. However, during the study period, if participants were found that they meet the excluded criteria. They would be excluded from the study.

The data of participants who were withdrawal or eliminated would be delete in the study.

\section{Plans to give access to the full protocol, participant level-data and statistical code $\{31 \mathrm{c}\}$}

The data/figures used and/or analyzed in this study are available from the corresponding author upon reasonable request. 


\section{Oversight and monitoring}

\section{Composition of the coordinating centre and trial steering committee $\{5 \mathrm{~d}\}$}

The Ethics Committee approved the study at the China-Japan Friendship Hospital of Jilin University, on October 30th 2019, approval number: (2019) Review No. (2019103004). The scientific design and implementation of the study will be carried out under the supervision and guidance of the Ethics Committee of the China-Japanese Union Hospital of Jilin University. The Ethics Committee will oversee the security and ethical aspects of the data. Authorized research doctors will collect data collection, and research assistants will assist in data collation and entry. All researchers involved in the project will receive unified questionnaire training to reduce data bias.

Research and data Collection Monitoring Group are responsible for contacting the researchers in order to inspect the facility and, as required, check various records of the clinical studies (for example, eCRF and other relevant data), provided that the confidentiality of the subjects is respected. During the study, inspectors are responsible for regular verification of eCRFs to verify compliance, completeness, accuracy and data consistency of the program, as well as compliance with relevant local clinical research regulations. The inspector should have the right to obtain the subject's medical records and other research-related records that need to be verified as entered in the eCRF. The researchers agreed to work with monitors to ensure that any problems found during these monitoring visits, including delays in the completion of the eCRF, were resolved.

\section{Composition of the data monitoring committee, its role and reporting structure $\{21 \mathrm{a}\}$}

Data monitoring committee (DMC) is composed of 2 professors and 2 attendance doctors. DMC will randomly audit $20 \%$ of the medical records and monitor the summary's quality to ensure that the overall variable accuracy is more than $98 \%$.

\section{Adverse event reporting and harms $\{22\}$}

Not applicatble.

\section{Frequency and plans for auditing trial conduct $\{23\}$}

Not applicatble. 


\section{Plans for communicating important protocol amendments to relevant parties (e.g. trial participants, ethical committees) $\{25\}$}

Any protocol modifications such as eligibility criteria, outcomes, analyses, et al. will be Submitted to Chinese Clinical Trail Registry (https://www.chictr.org.cn/), the Ethics Committee of Jilin University and the Journal for written approval. If any one above is not approved, no modification will be made.

\section{Dissemination plans $\{31$ a $\}$}

The result of this study will be submitted to an appropriate journal. All the data would upload to Chinese Clinical Trail Registry. The data/figures used and/or analyzed in this study are available from the corresponding author upon reasonable request.

\section{Discussion}

FFA is the substrate of myocardial energy metabolism. During AMI, the energy utilization of cardiomyocytes is impaired, which leads to the increase of intracellular FFA accumulation. At the same time, during the persistent state of a sympathetic storm, circulating FFA increases ${ }^{16}$. FFA also has many other important functions, including regulating energy metabolism, producing ATP, activating apoptosis signal pathway, etc ${ }^{17}$. Total plasma FFA levels are closely related to insulin resistance, obesity, and diabetes ${ }^{18}$. Huang et al. ${ }^{19}$ found that both cAMP and serum FFA increased in the model of ventricular tachycardia induced by ligation of the anterior descending branch in dogs. Similarly, in a rat model, after the isolated heart was ligated with catecholamine, it was found that FFA increased enzyme release, and decreased myocardial oxygen consumption by $20 \%{ }^{20}$. These animal studies show that serum FFA changes with $\mathrm{AMI}$ and sympathetic storm state, representing the state of myocardial energy metabolism to a certain extent. In clinical studies, the level of FFA is closely related to the increased risk of coronary heart disease ${ }^{21}$. At the same time, FFA is also one of the most sensitive indicators for early diagnosis of $\mathrm{AMI}$ and is a more sensitive marker than ECG measurement ${ }^{22}$. This finding is supported by the evidence that FFA reflects myocardial activity and energy metabolism. The increase in FFA level is an independent risk factor and independent diagnostic index of $\mathrm{AMI}$, but this result seems to have gender differences. Ying et al. ${ }^{3}$ found that the change of plasma FFA level after AMI was more significant in women than men. The relationship between FFA and ischemic heart disease is clear, but the role of predictive risk in patients with AMI is not clear. As mentioned earlier, the results of predictive studies on FFA and coronary heart disease (CHD) in the literature are not consistent. This may be different from the role of different FFA in the body. Yang et al. ${ }^{23}$ found that all FFAs except C14:0 were independent risk factors for $\mathrm{CHD}$ in non-diabetic patients. The ratio of EPA/AA is a possible new risk factor for coronary heart disease. The level of stearic acid may be related to blood coagulation and thrombosis ${ }^{24}$. These studies suggest that using total FFA alone to assess prognostic risk may overestimate the predictive ability of FFA. Therefore, we have designed a prospective, observational single-center study of AMI to determine plasma FFA in 
patients with acute ST-segment elevation. To systematically collect patient baseline data, we have designed a baseline questionnaire with the Epidemiological Survey team of the School of Public Health, Tianjin Medical University. It is worth noting that the plasma FFA level may be affected by the last mealtime. However, due to the acute onset of AMI, the time of arrival at the emergency department is uncontrollable. Therefore, it is hard to include the last mealtime in our clinical research because most patients cannot recall the exact time. As stated above, the plasma FFA level in the untreated disease state could represent the myocardial metabolic state. This limitation of the last mealtime does not prevent FFA from being an independent risk factor and valuable prognostic marker for AMI in CHD patients. At the same time, our questionnaire can also make a cross-sectional analysis of the factors affecting the prognosis of AMI. The purpose of this study is to explore the effects of different ages, gender, disease status, and onset time on plasma FFA. FFA is closely related to gender, age, and metabolic status. Although linoleic acid decreases slightly with age and the development of coronary heart disease, the composition spectrum of free fatty acids in a representative population is similar. Our exploratory study may be helpful to deepen the understanding of FFA.

\section{Trial Status}

This study protocol version number is 1.0, Date on 30th June, 2019. Recruitment began on 8th July, 2019. The approximate date when recruitment completed may be 31th, Dec, 2022.

\section{Abbreviations}

Acute ST-segment elevation myocardial infarction, AMI;

Free fatty acids, FFA;

Acute Coronary Syndrome, ACS;

Eicosapentaenoic acid, EPA;

a-linolenic acid, ALA;

Docosahexaenoic acid, DHA;

Arachidonic acid, AA;

high-performance liquid chromatography, HPLC;

European Society of Cardiology, ESC;

Electrocardiograph, ECG;

American College of Cardiology, ACC; 
American Heart Association, AHA;

Emergency Room, ER;

Docosatetraenoic Acid, DTA;

Docosapentaenoic Acid, DPA;

Data monitoring committee, DMC;

Standard Deviation, SD;

Hazzard ratio, HR;

Confidence Interval, $\mathrm{Cl}$.

\section{Declarations}

\section{Acknowledgements}

Not applicable

\section{Authors' contributions \{31b\}}

PY is the Chief Investigator; she conceived the study, led the proposal and protocol development. WC, SW and $X Y$ contributed to study design and to development of the proposal. HS was the lead trial methodologist. All authors read and approved the final manuscript.

\section{Funding $\{4\}$}

This study is founded by the project from Provincial Science and Technology Department, the grand number: 2019SC0012. The sponsor, Professor Ping Yang played a role in the design of the study, collection, analysis, and interpretation of the data and in writing the manuscript.

\section{Availability of data and materials $\{29\}$}

All the data would upload to Chinese Clinical Trail Registry. The data/figures used and/or analyzed in this study are available from the corresponding author upon reasonable request. 
Ethics approval and consent to participate $\{24\}$

The Ethics Committee approved the study at the China-Japan Friendship Hospital of Jilin University, on October 30th 2019, approval number: (2019) Review No. (2019103004).

\section{Consent for publication $\{32\}$}

Not applicable.

\section{Competing interests $\{28\}$}

The authors declare that they have no competing interests.

\section{Authors' information (optional)}

\section{References}

1. Gruzdeva O, Uchasova E, Dyleva Y, et al. Multivessel coronary artery disease, free fatty acids, oxidized LDL and its antibody in myocardial infarction. Lipids Health Dis Jul. 2014;9:13:111. doi:10.1186/1476-511x-13-111.

2. White HD, Chew DP. Acute myocardial infarction. Lancet Aug. 2008;16(9638):570-84. doi:10.1016/s0140-6736(08)61237-4. 372 ) .

3. Kan Y, Wang H, Lu J, Lin Z, Lin J, Gong P. Significance of plasma free fatty acid level for assessing and diagnosing acute myocardial infarction. Biomark Med Jun. 2020;14(9):739-47. doi:10.2217/bmm-2019-0291.

4. Carlsson M, Wessman Y, Almgren P, Groop L. High levels of nonesterified fatty acids are associated with increased familial risk of cardiovascular disease. Arterioscler Thromb Vasc Biol Jun. 2000;20(6):1588-94. doi:10.1161/01.atv.20.6.1588.

5. Sarafidis PA, Bakris GL. Non-esterified fatty acids and blood pressure elevation: a mechanism for hypertension in subjects with obesity/insulin resistance? J Hum Hypertens Jan. 2007;21(1):12-9. doi:10.1038/sj.jhh.1002103.

6. Charles MA, Fontbonne A, Thibult N, et al. High plasma nonesterified fatty acids are predictive of cancer mortality but not of coronary heart disease mortality: results from the Paris Prospective Study. Am J Epidemiol Feb. 2001;1(3):292-8. doi:10.1093/aje/153.3.292. 153 ) .

7. Schrieks IC, Nozza A, Stähli BE, et al. Adiponectin, Free Fatty Acids, and Cardiovascular Outcomes in Patients With Type 2 Diabetes and Acute Coronary Syndrome. Diabetes Care Aug. 2018;41(8):1792800. doi:10.2337/dc18-0158. 
8. Lázaro I, Rueda F, Cediel G, et al. Circulating Omega-3 Fatty Acids and Incident Adverse Events in Patients With Acute Myocardial Infarction. J Am Coll Cardiol Nov. 2020;3(18):2089-97. doi:10.1016/j.jacc.2020.08.073. 76 ) .

9. Lee SH, Shin MJ, Kim JS, et al. Blood eicosapentaenoic acid and docosahexaenoic acid as predictors of all-cause mortality in patients with acute myocardial infarction-data from Infarction Prognosis Study (IPS) Registry. Circ J Dec. 2009;73(12):2250-7. doi:10.1253/circj.cj-09-0327.

10. Ueeda M, Doumei T, Takaya Y, et al. Association of serum levels of arachidonic acid and eicosapentaenoic acid with prevalence of major adverse cardiac events after acute myocardial infarction. Heart Vessels Mar. 2011;26(2):145-52. doi:10.1007/s00380-010-0038-8.

11. Hicks KA, Tcheng JE, Bozkurt B, et al. 2014 ACC/AHA Key Data Elements and Definitions for Cardiovascular Endpoint Events in Clinical Trials: A Report of the American College of Cardiology/American Heart Association Task Force on Clinical Data Standards (Writing Committee to Develop Cardiovascular Endpoints Data Standards). J Am Coll Cardiol Jul 28 2015;66(4):403-69. doi:10.1016/j.jacc.2014.12.018.

12. Ibanez B, James S, Agewall S, et al. 2017 ESC Guidelines for the management of acute myocardial infarction in patients presenting with ST-segment elevation: The Task Force for the management of acute myocardial infarction in patients presenting with ST-segment elevation of the European Society of Cardiology (ESC). Eur Heart J Jan. 2018;7(2):119-77. doi:10.1093/eurheartj/ehx393. 39 )

13. Liosis S, Bauer T, Schiele R, et al. Predictors of 1-year mortality in patients with contemporary guideline-adherent therapy after acute myocardial infarction: results from the OMEGA study. Clin Res Cardiol Sep. 2013;102(9):671-7. doi:10.1007/s00392-013-0581-2.

14. Li J, Li X, Wang Q, et al. ST-segment elevation myocardial infarction in China from 2001 to 2011 (the China PEACE-Retrospective Acute Myocardial Infarction Study): a retrospective analysis of hospital data. The Lancet. 2015;385(9966):441-51. doi:10.1016/s0140-6736(14)60921-1.

15. Hsieh FY, Lavori PW. Sample-size calculations for the Cox proportional hazards regression model with nonbinary covariates. Control Clin Trials Dec. 2000;21(6):552-60. doi:10.1016/s01972456(00)00104-5.

16. Lommi J, Kupari M, Yki-Järvinen $\mathrm{H}$. Free fatty acid kinetics and oxidation in congestive heart failure. Am J Cardiol Jan. 1998;1(1):45-50. doi:10.1016/s0002-9149(97)00804-7. 81 ) .

17. Taegtmeyer $\mathrm{H}$, McNulty P, Young ME. Adaptation and maladaptation of the heart in diabetes: Part I: general concepts. Circulation Apr. 2002;9(14):1727-33. doi:10.1161/01.cir.0000012466.50373.e8. $105)$.

18. Pankow JS, Duncan BB, Schmidt MI, et al. Fasting plasma free fatty acids and risk of type 2 diabetes: the atherosclerosis risk in communities study. Diabetes Care Jan. 2004;27(1):77-82. doi:10.2337/diacare.27.1.77.

19. Huang DX, Dong J. [Cyclic AMP levels in the plasma and ischemic myocardium and free fatty-acid levels in the serum following coronary artery ligation in dogs: relation to ventricular fibrillation]. 
Zhonghua Nei Ke Za Zhi Aug. 1989;28(8):457-9, 507-8.

20. Opie LH. Sympathetic stimulation of ischemic myocardium: role of plasma free fatty acids and potassium. J Cardiovasc Pharmacol. 1988;12(Suppl 1):31-8.

21. Pirro $M$, Mauriège $P$, Tchernof $A$, et al. Plasma free fatty acid levels and the risk of ischemic heart disease in men: prospective results from the Québec Cardiovascular Study. Atherosclerosis Feb. 2002;160(2):377-84. doi:10.1016/s0021-9150(01)00588-3.

22. Kleinfeld AM, Prothro D, Brown DL, Davis RC, Richieri GV, DeMaria A. Increases in serum unbound free fatty acid levels following coronary angioplasty. Am J Cardiol Dec. 1996;15(12):1350-4. doi:10.1016/s0002-9149(96)00651-0. 78 ) .

23. Yang N, Guo S, Zheng F, Gan X, Ning L, Dong S. High plasma fatty acid concentrations were present in non-diabetic patients with coronary heart disease. Clin Lab. 2014;60(1):125-31. doi:10.7754/clin.lab.2013.130108.

24. Hoak JC. Stearic acid, clotting, and thrombosis. Am J Clin Nutr Dec. 1994;60(6 Suppl):1050s-1053s. doi:10.1093/ajen/60.6.1050S.

\section{Tables}

Table 1 Power and Sample Size calculation Cox Regression

Numeric Results for FFA

\begin{tabular}{lrcccccr}
\hline & & & \multicolumn{5}{c}{ R-Squared } \\
& Sample & Reg. & S.D. & Event & X1 vs & Two- & \\
& Size & Coef. & of X1 & Rate Other X's & Sided & \\
Power & $(\mathrm{N})$ & $(\mathrm{B})$ & $(\mathrm{SD})$ & $(\mathrm{P})$ & $(\mathrm{R} 2)$ & Alpha & Beta \\
0.80049 & 597 & 0.1130 & 7.3660 & 0.0190 & 0.0000 & 0.05000 & 0.19951
\end{tabular}

Dropout-Inflated Sample Size

Dropout-

Inflated Expected

Enrollment Number of

Sample Size Sample Size Dropouts

Dropout Rate $\mathrm{N}$ $\mathrm{N}^{\prime}$ 597 747
D 150

Numeric Results for EPA

\begin{tabular}{lrcccrrr}
\hline & & & \multicolumn{5}{c}{ R-Squared } \\
& Sample & Reg. & S.D. & \multicolumn{2}{c}{ Event } & X1 vs & Two- \\
Size & Coef. & of X1 & Rate & Other X's & Sided & \\
Power & $(\mathrm{N})$ & $(\mathrm{B})$ & $(\mathrm{SD})$ & $(\mathrm{P})$ & $(\mathrm{R} 2)$ & Alpha & Beta \\
0.81117 & 18 & -0.2700 & 18.0000 & 0.0190 & 0.0000 & 0.05000 & 0.18883 \\
\multicolumn{2}{l}{ Dropout-Inflated Sample Size } & & & & &
\end{tabular}


Dropout-

Inflated Expected

Enrollment Number of

Dropout Rate

Sample Size

Sample Size

Dropouts

$20 \%$

$\mathrm{N}$

$\mathrm{N}^{\prime}$

D

18

23

5

Numeric Results for ALA

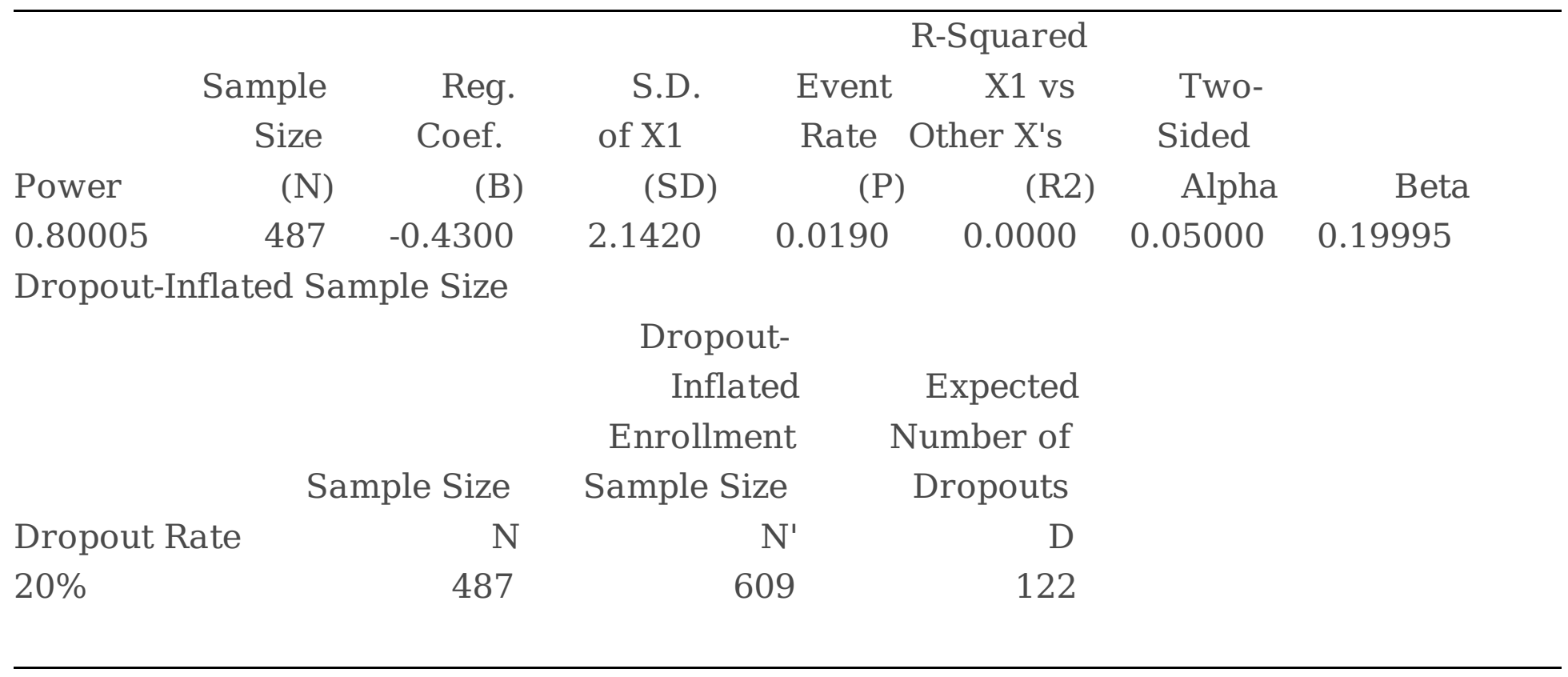

Report Definitions

Power is the probability of rejecting a false null hypothesis. It should be close to one.

$\mathrm{N}$ is the size of the sample drawn from the population.

$\mathrm{B}$ is the size of the regression coefficent to be detected

$\mathrm{SD}$ is the standard deviation of $\mathrm{X} 1$.

$\mathrm{P}$ is the event rate.

$\mathrm{R} 2$ is the R-squared achieved when $\mathrm{X} 1$ is regressed on the other covariates.

Alpha is the probability of rejecting a true null hypothesis.

Beta is the probability of accepting a false null hypothesis.

Dropout Rate (DR) is the percentage of subjects (or items) that are expected to be lost at random during the course of the study and for whom no response data will be collected (i.e. will be treated as "missing"). $\mathrm{N}$ is the evaluable sample size at which power is computed. If $\mathrm{N}$ subjects are evaluated out of the $\mathrm{N}^{\prime}$ subjects that are enrolled in the study, the design will achieve the stated power. $\mathrm{N}^{\prime}$ is the total number of subjects that should be enrolled in the study in order to end up with $\mathrm{N}$ evaluable subjects, based on the assumed dropout rate. After solving for $N, N^{\prime}$ is calculated by inflating $N$ using the formula $N^{\prime}=N /(1-D R)$, with $\mathrm{N}^{\prime}$ always rounded up. 
Figures

\begin{tabular}{|c|c|c|c|c|c|c|}
\hline \multirow[b]{3}{*}{ Timepoint } & \multicolumn{6}{|c|}{ STUDY PERIOD } \\
\hline & \multirow{2}{*}{$\begin{array}{c}\text { Enrolment } \\
2019.07- \\
2022.12 \\
\end{array}$} & \multirow{2}{*}{$\begin{array}{c}\text { Allocation } \\
2019.09- \\
2022.12 \\
\end{array}$} & \multicolumn{3}{|c|}{ Post-allocation } & \multirow{2}{*}{$\begin{array}{c}\text { Close-out } \\
2023.1- \\
2023.12 \\
\end{array}$} \\
\hline & & & $2 m$ & $6 m$ & $12 m$ & \\
\hline \multicolumn{7}{|l|}{ ENROLMENT: } \\
\hline \multirow{2}{*}{$\begin{array}{l}\text { Eligibility screen } \\
\text { Informed consent }\end{array}$} & $\mathrm{X}$ & & & & & \\
\hline & $\mathrm{X}$ & & & & & \\
\hline \multirow{2}{*}{$\begin{array}{l}\text { FFA test } \\
\text { Baseline information } \\
\text { acquired }\end{array}$} & $\mathrm{X}$ & & & & & \\
\hline & $\mathrm{x}$ & & & & & \\
\hline Allocation & & $\mathrm{x}$ & & & & \\
\hline ASSESSMENTS: & & & $\mathrm{x}$ & & & \\
\hline Follow up & & & & & $\rightarrow$ & \\
\hline Major endpoint event & & & $\mathrm{X}$ & $\mathrm{X}$ & $\mathrm{X}$ & $\mathrm{X}$ \\
\hline Other endpoint event & & & $x$ & $x$ & $x$ & $x$ \\
\hline
\end{tabular}

Figure 1

Participant timeline of the study

\section{Supplementary Files}

This is a list of supplementary files associated with this preprint. Click to download.

- SupplementaryMaterial.docx 